\title{
Una tarea para la escuela de negocios del siglo XXI: estudiantes computacionalmente competentes \\ A task for business school in the 21 st century: competent computationally students
}

\author{
M. Consuelo Calafat ${ }^{1}$, Ana Debón ${ }^{2}$, M. del Mar Marín ${ }^{1}$, Rosa Puertas ${ }^{1}$, F. Javier Ribal ${ }^{1}$ \\ macamar3@esp.upv.es, andeau@eio.upv.es,mmarins@esp.upv.es, rpuertas@esp.upv.es, frarisan@esp.upv.es \\ ${ }^{1}$ Departamento de Economía y Ciencias Sociales, \\ Facultad de Administración y Dirección de \\ Empresas \\ Universitat Politècnica de València \\ Valencia, España \\ ${ }^{2}$ Departamento de Estadística e I.O. Aplicadas y \\ Calidad \\ Facultad de Administración y Dirección de \\ Empresas \\ Universitat Politècnica de València \\ Valencia, España
}

\begin{abstract}
Resumen- El mercado laboral de los estudiantes de administración y dirección de empresas exige conocimientos informáticos que deben prepararse en las escuelas de negocios. Las escuelas de negocios deben identificar las herramientas y habilidades esenciales necesarias para el desarrollo de la profesión en el siglo XXI. En este contexto, la Facultad de Administración y Dirección de Empresas de la Universitat Politècnica de València hace que los estudiantes de grado estén siempre en contacto con la tecnología y las herramientas informáticas adecuadas para cada asignatura. El objetivo de este estudio es doble. En primer lugar, este estudio tiene como objetivo recoger los conocimientos técnicos en las herramientas informáticas de la formación en el proceso de aprendizaje del grado en Gestión y Administración Pública (GAP). En segundo lugar, analizar si este conocimiento se ajusta a las demandas del sector público y privado.
\end{abstract}

Palabras clave: Competencias computacionales, Administración Pública, Software.

Abstract- The labor market for business and management students demands computer skills that must be prepared in business schools. Business schools should identify the essential tools and skills necessary for the development of the profession. In this context, the Faculty of Business Administration and Management at the Universitat Politècnica de València means that degree students are always in contact with the appropriate technology and computer tools for each subject. The aim of this study is two-fold. Firstly, this study aims to collect the technical knowledge in the computer tools of the training in the degree on Gestión y Administración Pública (GAP) students learning process. Secondly, to analyze whether this knowledge fits the public and private sector demands.

\section{Keywords: Computational skills, Public Administration, Software}

\section{INTRODUCCIÓN}

La mejora de la empleabilidad de los egresados en un contexto académico se busca adecuando la capacitación por competencias que enfaticen la adquisición de habilidades prácticas para el desempeño óptimo en el ámbito laboral (Ramirez-Martinell, 2010). Sin embargo, hay un diferencial existente entre las habilidades aprendidas por los estudiantes y la demanda laboral existente y así lo ponen de manifiesto autores como Cappelli (2014), entre otros, aportando evidencias de ello en Estados Unidos.

En un intento por actualizar los contenidos y hacerlos más prácticos en las distintas materias que componen los planes docentes de los actuales grados se introduce el manejo de determinados programas informáticos. Estos programas son una herramienta de apoyo para el aprendizaje y resolución de casos aplicados. Y además, en sí mismos, potentes instrumentos que facilitan la aplicación de conocimientos, pero su manejo adecuado requiere de una completa capacitación del alumno y de una continua actualización.

Por otra parte, en la actualidad los universitarios son nativos digitales, con acceso a herramientas informáticas que utilizan y manejan con gran destreza (Sánchez y Castro, 2013). Por lo que introducir en las asignaturas el aprendizaje de software permite aprovechar este potencial. Sin embargo, según señala Oliver et al. (2000) evaluar adecuadamente esas competencias y adaptarlos al mercado laboral supone un gran esfuerzo por parte de las escuelas de negocios. Pues todo ello, supone definir un conjunto de habilidades que describan adecuadamente a una persona con conocimientos de informática, así como la necesidad de diseñar herramientas de evaluación para medir los niveles de estas habilidades.

En la Facultad de Administración y Dirección de Empresas (FADE) de la Universitat Politècnica de València (UPV) se han concretado las destrezas informáticas adquiridas por los alumnos del Grado en Gestión y Administración Pública (GGAP) para su adecuación a las exigencias del mercado laboral de acuerdo a los expertos profesionales que forman el comité asesor de la Facultad. Con este objetivo se ha desarrollado el proyecto institucional TALiGAP considerando 
la definición de alfabetización informática ("computer literacy”) según Simonson et al (1987):

"Una comprensión de las características, capacidades y aplicaciones de la computadora, así como la capacidad de implementar este conocimiento en el uso hábil y productivo de aplicaciones informáticas adecuadas para roles individuales en la sociedad. (pág. 232)" y consideramos su evaluación y adecuación al mundo laboral.

\section{CONTEXTO}

La UPV consciente de la gran importancia de las competencias transversales en el desempeño profesional de los titulados universitarios ha desarrollado un proyecto institucional (Universitat Politècnica de València, 2021)) con 13 competencias Transversales. Estas competencias se han introducido en todos los Grados y Másteres, siendo necesario el aprendizaje y la evaluación continua de todas ellas.

Concretamente, el proyecto institucional TALiGAP se articula sobre la competencia 13, "Instrumental específica", entendida como la capacidad de utilizar las técnicas, habilidades y herramientas actualizadas necesarias para la práctica de la profesión. Esta competencia hace referencia al uso de las tecnologías necesarias para el ejercicio profesional asociado a cada titulación, en este caso al estudiante de Grado en Gestión y Administración Pública (GGAP) y de acuerdo a Ribal et al (2019) el objetivo de la misma debe ser aproximar las competencias y habilidades de los graduados a los requerimientos y demandas de las empresas. GGAP contempla en su plan de estudios en amplio abanico de asignaturas que incorporan prácticas en las cuales se hace uso de un determinado software (Facultad Administración y Dirección de Empresas, 2021).

Para el desarrollo de este proyecto, en primer lugar, se asignaron los contenidos a la competencia transversal 13 en GGAP, realizando un inventario del talento informático de nuestros titulados. Los resultados se han publicado en Baviera et al. (2020) de forma detallada y suponen dos itinerarios definidos uno en ofimática y otro en estadística.

Posteriormente, se desarrolló una conexión interdisciplinar de cada herramienta informática utilizada y se crearon rúbricas para evaluar la competencia transversal 13 para cada itinerario, y que sirva tanto de diagnóstico del grado de desarrollo por parte del alumnado como de referencia para la práctica profesional que determinan los profesionales en el ejercicio de su actividad. Esta fase permitirá corregir las deficiencias e integrarlas de forma coordinada con las asignaturas a lo largo del grado. La implantación definitiva de la competencia permitirá certificar e integrar los conocimientos de cada software en el expediente académico del estudiante.

Para validar estas rubricas y hacerlas compatibles con la demanda real se ha definido una metodología de trabajo que permite recoger información para el diagnóstico inicial y la corrección de las deficiencias.

\section{DESCRIPCIÓN}

El estudio sobre el desarrollo de las competencias digitales alcanzadas por los alumnos de GGAP se ha realizado a través de rúbricas con los profesores que utilizan herramientas de office y a expertos vinculados a la Facultad de ADE. A los profesores del grado se les preguntada sobre el nivel de competencia alcanzado por los alumnos en las herramientas office. A los expertos se les preguntaba sobre el nivel de conocimiento de acuerdo con la necesidad de estas herramientas en su empresa o en su trabajo en la administración pública. El estudio se ha llevado a cabo en el curso 20-21 por ello las opiniones de los profesores y expertos se basan en su experiencia este curso aunque los resultados hacen inferencia sobre todos los estudiantes de GGAP a pesar de las limitaciones que supone el estudio en un momento concreto y con unos profesores determinados.

En la Figura 1 se resume el proceso seguido. Se recopiló la información de las asignaturas en las que se requiere la utilización de programas office por parte del alumno $y$, paralelamente, se crearon rúbricas para cada uno de los programas ofimáticos, tanto de la versión Microsoft como en libre office (Word/Writer, PowerPoint/Impress, Excel/Calc). Posteriormente se pasaron las rúbricas a los profesores para conocer su opinión sobre el nivel alcanzado por el alumnado de cada punto considerado en la rúbrica: No Alcanzado (N/A); En Desarrollo (E/D); Bueno (BU); Excelente (EX). Además, se enviaron las rubricas a expertos relacionados con el grado para conocer su opinión sobre la utilización de los programas informáticos y considerar si en su empresa o administración pública es necesario saber realizar las tareas indicadas en la rúbrica y cuál es el nivel de necesidad: Nada Necesario $(\mathrm{N} / \mathrm{N})$; Poco Necesario (P/N); Necesario (N); Muy Necesario (M/N). Finalmente, los resultados obtenidos se muestran a los profesores para coordinar acciones de mejora en los puntos donde se han detectado deficiencias entre niveles alcanzados por los alumnos y las necesidades manifestadas por los expertos.
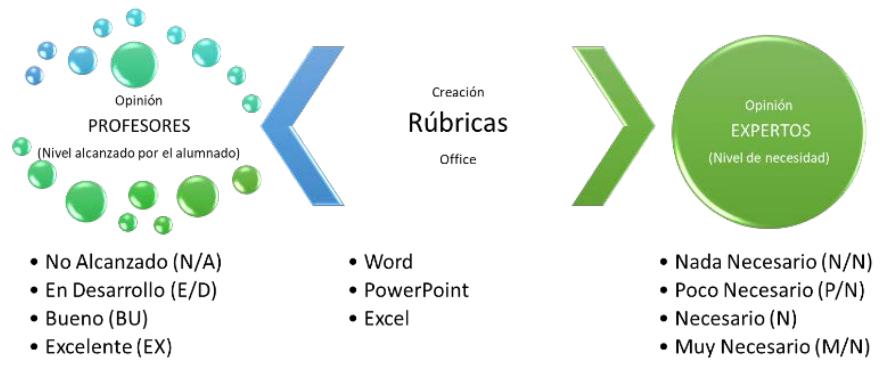

Figura 1. Flujo de trabajo seguido

\section{RESUltados}

Los alumnos de GGAP utilizan programas de ofimática en asignaturas desde primero hasta cuarto curso. En total se utilizan programas office en 14 asignaturas con 76,5 créditos ( $32 \%$ de total de créditos del Grado). Los resultados obtenidos para cada uno de los programas estudiados se detallan en las subsecciones siguientes.

4.1 Resultados obtenidos para Word/Writer

Para evaluar las habilidades de los alumnos en Word/Writer contestaron 6 profesores de distintas asignaturas y para evaluar el grado de necesidad contestaron 10 expertos.

Los alumnos utilizan Word/Writer desde el primer semestre de primero. Los resultados de los profesores que han participado en la rúbrica muestran, en términos generales, un nivel bueno, excepto en dar formato a los gráficos e insertar SmartArt. 
Tras analizar la distribución de los item en cada una de las rubricas y comparar opiniones de profesores y expertos tal y como se muestra a modo de ejemplo en la Figura 2 para un item de la rúbrica de Word.

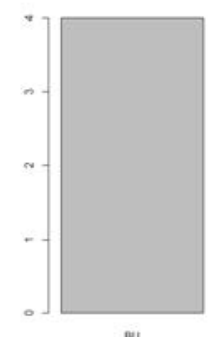

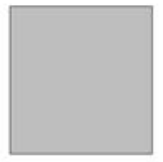
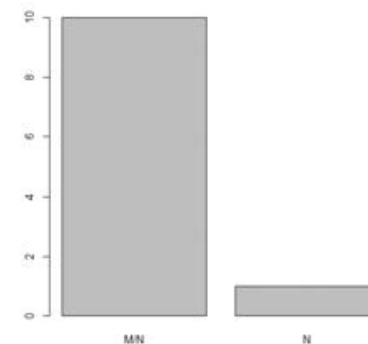

Figura 2. Opiniones de los profesores y expertos respecto del nivel alcanzado en los estudiantes en Word en el item "crear un documento".

Los resultados más frecuentes en cada punto de la rúbrica son los siguientes:

- Crear y administrar documentos:

- Crear un documento: Bueno

- Navegar por un documento: Bueno

- Dar formato a un documento: Excelente

- Personalizar opciones y vistas para los documentos: En desarrollo

- Imprimir y guardar documentos: En desarrollo

Dar formato al texto, párrafos y secciones

- Insertar texto y párrafos: Bueno

- Dar formato a texto y párrafos: Bueno

- Ordenar y agrupar texto y párrafos: Bueno

- Crear tablas y listas

- Crear una tabla: Bueno

- Modificar una tabla: Bueno

- Crear y modificar una lista: Bueno

- Crear y administrar referencias

- Crear y administrar marcadores de referencia: Bueno

- Crear y administrar referencias simples: Bueno

- Insertar elementos gráficos y darles formato

- Insertar elementos gráficos: Bueno

- Dar formato a elementos gráficos: En desarrollo

- Insertar y dar formato a elementos gráficos SmartArt: En desarrollo.

Los resultados obtenidos con las respuestas de los expertos muestran que, en general, consideran muy necesaria la utilización de Word para realizar tareas en las empresas o administraciones públicas, excepto en dar formato a los gráficos e insertar SmartArt.

Los resultados en cada punto de la rúbrica son los siguientes:

- Crear y administrar documentos:

- Crear un documento: Muy Necesario

- Navegar por un documento: Muy Necesario

- Dar formato a un documento: Muy Necesario

- Personalizar opciones y vistas para los documentos: Necesario
- Imprimir y guardar documentos: Muy Necesario

- Dar formato al texto, párrafos y secciones

- Insertar texto y párrafos: Muy Necesario

- Dar formato a texto y párrafos: Necesario

- Ordenar y agrupar texto y párrafos: Necesario-Muy Necesario

- Crear tablas y listas

- Crear una tabla: Muy Necesario

- Modificar una tabla: Muy Necesario

- Crear y modificar una lista: Necesario -Muy Necesario

- Crear y administrar referencias

- Crear y administrar marcadores de referencia: Necesario

- Crear y administrar referencias simples: Necesario

Insertar elementos gráficos y darles formato

- Insertar elementos gráficos: Necesario

- Dar formato a elementos gráficos: No está clara la necesidad

- Insertar y dar formato a elementos gráficos SmartArt: No está clara la necesidad

4.2. Resultados obtenidos para PowerPoint/Impress

El Power Point se utiliza menos que el Word, en concreto en una asignatura en segundo, una de tercero y una de cuarto. Por tanto, han contestado 3 profesores a las rúbricas definidas. Las respuestas recogidas de los expertos del comité asesor corresponden a 8 expertos.

Los resultados de los profesores muestran que, en términos generales, el nivel es bueno, excepto en administrar varias presentaciones que está en desarrollo.

Los resultados para cada punto de la rúbrica son los siguientes: - Crear una presentación

- Insertar y dar formato a diapositivas: Bueno

- Modificar diapositivas, documentos y notas: Bueno

- Ordenar y agrupar diapositivas Bueno

- Cambiar las opciones y vistas de presentación: Bueno

- Configurar una presentación para su impresión: No alcanzado

- Configurar y realizar una presentación de diapositivas: Bueno

- Insertar y dar formato a texto, formas e imágenes

- Insertar y dar formato a texto: Bueno

- Insertar y dar formato a formas y cuadros de texto: Bueno

- Insertar y dar formato a imágenes: Bueno

- Ordenar y agrupar objetos: Bueno

- Insertar tablas, gráficos, elementos gráficos SmartArt y medios

- Insertar y dar formato a tablas: Bueno

- Insertar y dar formato a gráficos: Bueno

- Insertar y dar formato a elementos gráficos SmartArt: En desarrollo

- Insertar y administrar medios: No alcanzado

Aplicar transiciones y animaciones

- Aplicar transiciones de diapositivas: En desarrollo 
- Animar contenido de diapositiva: En desarrollo

- Configurar tiempos de transiciones y animaciones: En desarrollo

- Administrar varias presentaciones

- Combinar contenido de varias presentaciones: En desarrollo

- Finalizar las presentaciones: En desarrollo

El comité asesor considera que, en términos generales, las tareas para crear una presentación son muy necesarias y las tareas de formatear texto y formas son necesarias, así como insertar tablas y gráficos. Las tareas animaciones y SmartArt no se consideran tan necesarias.

Los resultados para cada uno de los ítems de la rúbrica son los siguientes:

\section{- Crear una presentación}

- Crear una presentación: Muy Necesario

- Insertar y dar formato a diapositivas: Necesario - Muy Necesario

- Modificar diapositivas, documentos y notas: Muy Necesario

- Ordenar y agrupar diapositivas: Necesario

- Cambiar las opciones y vistas de presentación: Muy Necesario

- Configurar una presentación para su impresión: Muy Necesario

- Configurar y realizar una presentación de diapositivas: Necesario

- Insertar y dar formato a texto, formas e imágenes

- Insertar y dar formato a texto: Muy Necesario

- Insertar y dar formato a formas y cuadros de texto: Muy Necesario

- Insertar y dar formato a imágenes: Muy Necesario

- Ordenar y agrupar objetos: Poco necesario

Insertar tablas, gráficos, elementos gráficos SmartArt y medios

- Insertar y dar formato a tablas: Necesario

- Insertar y dar formato a gráficos: Necesario

- Insertar y dar formato a elementos gráficos SmartArt: Poco necesario

- Insertar y administrar medios: Nada necesario Aplicar transiciones y animaciones

- Aplicar transiciones de diapositivas: Poco necesario

- Animar contenido de diapositiva: Poco necesario

- Configurar tiempos de transiciones y animaciones: Nada necesario

- Administrar varias presentaciones

- Combinar contenido de varias presentaciones: Poco necesario

- Finalizar las presentaciones: Poco necesario

\subsection{Resultados obtenidos para Excel/Calc}

Los alumnos utilizan Excel en prácticamente todos los semestres desde el primer semestre de primero y hasta cuarto. Por tanto, Excel es el programa más utilizado del paquete office.
Las rúbricas para la evaluación del nivel alcanzado han sido contestadas por 7 profesores y por parte del comité asesor por 8 expertos.

Los profesores, en términos generales, indican que los alumnos de GAP no tienen formación suficiente para realizar pruebas sobre el talento en Excel.

A continuación, se resumen el nivel alcanzado en cada uno de los ítems:

- Crear y administrar hojas de cálculo y libros

- Crear hojas de cálculo y libros: Bueno

- Navegar a través de hojas de cálculo y libros: Bueno

- Dar formato a hojas de cálculo y libros: Bueno

- Personalizar opciones y vistas en hojas de cálculo y libros: No alcanzado

- Configurar hojas de cálculo y libros para su distribución: No alcanzado

- Administrar celdas de datos e intervalos

- Insertar datos en celdas e intervalos: En desarrollo

- Dar formato a celdas e intervalos: Bueno

- Resumir y organizar los datos: En desarrollo

- Crear tablas

- Creación y administración de tablas: No alcanzado

- Administrar estilos de tabla y opciones: No alcanzado

- Filtrar y ordenar una tabla: No alcanzado

- Realizar operaciones con fórmulas y funciones

- Resumir datos con funciones: En desarrollo

- Realizar operaciones condicionales con funciones: No alcanzado

- Dar formato y modificar el texto con funciones: No alcanzado

- Formulas avanzadas: No alcanzado

- Tablas dinámicas: No alcanzado

- Crear gráficos y objetos

- Crear gráficos: Bueno

- Dar formato a elementos gráficos: Bueno

- Insertar y dar formato a objetos: En desarrollo

En la asignatura de Técnicas y Métodos de $4^{\circ}$ realizan prácticas utilizando programación lineal en Excel y los alumnos alcanzan un nivel excelente.

Los resultados obtenidos con las respuestas del comité asesor muestran que, en términos generales, las tareas de Excel son necesarias o muy necesarias.

Los resultados obtenidos para cada ítem son los siguientes:

- Crear y administrar hojas de cálculo y libros

- Crear hojas de cálculo y libros: Muy Necesario

- Navegar a través de hojas de cálculo y libros:

Necesario - Muy Necesario

- Dar formato a hojas de cálculo y libros: Muy Necesario

- Personalizar opciones y vistas en hojas de cálculo y libros: Necesario

- Configurar hojas de cálculo y libros para su distribución: Necesario - Muy Necesario

- Administrar celdas de datos e intervalos 
- Insertar datos en celdas e intervalos: Necesario

- Dar formato a celdas e intervalos: Necesario - Muy Necesario

- Resumir y organizar los datos: Necesario

\section{Crear tablas}

- Creación y administración de tablas: Muy Necesario

- Administrar estilos de tabla y opciones: Necesario Muy Necesario

- Filtrar y ordenar una tabla: Necesario - Muy Necesario

- Realizar operaciones con fórmulas y funciones

- Resumir datos con funciones: Necesario

- Realizar operaciones condicionales con funciones: Necesario

- Dar formato y modificar el texto con funciones: Necesario

- Formulas avanzadas: No está clara la necesidad

- Tablas dinámicas: No está clara la necesidad

\section{- Crear gráficos y objetos}

- Crear gráficos: Muy Necesario

- Dar formato a elementos gráficos: Necesario

- Insertar y dar formato a objetos: No está clara la necesidad

\section{CONCLUSIONES}

El mercado laboral demanda el uso de las tecnologías necesarias para el ejercicio profesional, por ello es de vital importancia la adecuación y evaluación de dicha competencia transversal que se contempla en el proyecto institucional de la UPV. Así pues, en la FADE de la UPV se ha realizado un diagnóstico y adecuación de contenidos relativos al uso de software en GGAP y de acuerdo con profesionales que forman su comité asesor. Los resultados muestran que en los programas de procesamiento de texto y presentaciones coincide el grado de competencia alcanzado por los alumnos y las necesidades que indican los expertos. En cambio, en los programas de cálculo hay importantes diferencias. Los alumnos alcanzan niveles buenos en acciones como la creación y administración de hojas de cálculo y libros y en la creación de gráficos y objetos. Estos epígrafes son considerados necesarios para los expertos. En cambio, los alumnos no alcanzan niveles adecuados en las tareas para la creación de tablas y en la realización de operaciones con fórmulas y funciones, aunque hay una asignatura en la que realizan programación lineal con el complemento de Excel Solver. Los expertos consideran estas tareas necesarias para el desempeño de las funciones en las empresas o administraciones públicas. Estos resultados están en línea con los obtenidos por Formby et al (2017) que sugieren que las necesidades y oportunidades para las habilidades analíticas basadas en Excel son omnipresentes en las empresas de todos los tamaños y omnipresente en los negocios.

Aunque no se ha preguntado por el nivel de competencia adquirido en los programas de bases de datos, los alumnos realizan 10 horas de prácticas para la creación y relación de tablas, diseño de formularios, programación de consultas e informes.

Para ello, se propone informar a los profesores que utilizan herramientas informáticas de las deficiencias de los alumnos principalmente en la hoja de cálculo según las opiniones de los expertos.

Por otra parte, se propone que se impulse desde Decanato la utilización en las aulas de programas de ofimática libres, como OpenOffice o LibreOffice, siguiendo la línea de algunas asignaturas que ya lo utilizan, como es el caso de la asignatura Informática Aplicada que se imparte en el primer semestre de primero. Pues de acuerdo con Tickle et al. (2014) el papel de las universidades para preparar los estudiantes en estas habilidades resulta fundamental.

\section{AGRADECIMIENTOS}

Este trabajo ha sido realizado gracias a la financiación del Proyecto de Innovación y Mejora Educativa (PIME/18-19/60) de la convocatoria Aprendizaje + Docencia de la Universitat Politècnica de València.

Los autores agradecen los comentarios realizados por los dos revisores que han mejorado significativamente nuestro trabajo.

\section{REFERENCIAS}

Baviera, A, Babiloni, E., Debón, A., Marín, M., Puertas, R., Ribal, J., Skorczynska, H. \& Vallada, E. (2021, April). Inventario de talento informático en GADE y GGAP. In IN-RED 2020: VI Congreso de Innovación Educativa y Docencia en Red (pp. 1025-1033). Editorial Universitat Politècnica de València.

Cappelli, P. (2014). Skill gaps, skill shortages and skill mismatches: Evidence for the US (No. w20382). National Bureau of Economic Research.

Facultad Administración y Dirección de Empresas. (8 de agosto de 2021) Grado GAP. https://www.upv.es/entidades/ADE/infoweb/fade/info/63 0193normalc.html.

Formby, S. K., Medlin, D., \& Ellington, V. B. (2017). Microsoft Excel ${ }^{\circledR}$ : is it an important job skill for college graduates?. Information Systems Education Journal, 15(3), 55.

Oliver, R., Towers, S., \& Oliver, H. (2000). Information and Communications Technology Literacy-Getting serious about IT. In EdMedia+ Innovate Learning (pp. 862-867). Association for the Advancement of Computing in Education (AACE).

Ramirez-Martinell, A. (2009). Educational Video: Exploring the complex relationship between production, educational use and audience (Doctoral dissertation, Lancaster University).

Ribal Sanchis, F. J., Andrés González-Moralejo, S., Blasco Ruiz, A., Cervelló Royo, R. E., \& Chirivella González, V. (2019). La competencia instrumental específica en el grado de Administración y Dirección de Empresas de la Universitat Politècnica de València. JIDDO. I Jornada de innovación en docencia universitaria para la dirección de organizaciones públicas y privadas, 101-1096.

Sánchez Espinoza, A., \& Castro Ricalde, D. (2013). Cerrando la brecha entre nativos e inmigrantes digitales a través de las competencias informáticas e informacionales. Apertura 16 (1), pp 6-15. 
Tickle, L., Kyng, T., \& Wood, L. N. (2014). The role of universities in preparing graduates to use software in the financial services workplace. International Journal of Mathematical Education in Science and Technology, 45(2), 200-213.
Universitat Politècnica de València. ( 8 de agosto de 2021) Competencias http://www.upv.es/contenidos/COMPTRAN/
Transversales.

政

